\title{
INFLUÊNCIA DE VARIÁVEIS CONTEXTUAIS EM MEDIDAS NÃO-PARAMÉTRICAS DE EFICIÊNCIA: UMA APLICAÇÃO COM MÉTODOS DE REAMOSTRAGEM
}

\author{
Mirian Oliveira de Souza \\ Empresa Brasileira de Pesquisa Agropecuária (Embrapa) \\ Brasília - DF, Brasil \\ mirian.souza@embrapa.br \\ Geraldo da Silva e Souza \\ Dep. de Estatística - Universidade de Brasília (UnB) \\ Brasília - DF, Brasil \\ geraldo.souza@embrapa.br

\section{Roberta Blass Staub*} \\ Dep. de Estudos e Pesquisas - Banco Central do Brasil \\ Brasília - DF, Brasil \\ roberta.blass@bcb.gov.br \\ * Corresponding author / autor para quem as correspondências devem ser encaminhadas \\ Recebido em 06/2007; aceito em 04/2009 após 1 revisão \\ Received June 2007; accepted April 2009 after one revision
}

\section{Resumo}

Avaliam-se três técnicas do tipo bootstrap para a análise estatística de um modelo não-paramétrico de produção em que uma medida de eficiência DEA é afetada potencialmente por um conjunto de fatores exógenos. A aplicação de interesse diz respeito a avaliação da significância das variáveis contextuais receita própria, melhoria de processos, intensidade de parcerias, tipo e tamanho nas eficiências técnicas de produção dos centros de pesquisa da Embrapa. Conclui-se que o bootstrap associado ao estimador de máxima verossimilhança produz o melhor ajuste do ponto de vista da correlação de Pearson entre valores observados e preditos e é o mais informativo relativamente a significância das variáveis consideradas. Com exceção de tamanho todas as variáveis contextuais são estatisticamente significantes. Receita própria, melhoria de processos e intensidade de parcerias são todas positivamente associadas com a eficiência técnica.

Palavras-chave: DEA; bootstrap; estimação em dois estágios.

\begin{abstract}
We evaluate three bootstrap techniques for the statistical analysis of a non parametric production model for which a DEA measure of efficiency is potentially affected by a set of exogenous factors. The application of interest relates to the assessment of the significance of the contextual variables income generation, processes improvement, intensity of partnerships, type and size on the technical efficiencies of Embrapa's research centers. It is concluded that the bootstrap of the maximum likelihood estimator provides the best fit from the point of view of Pearson correlation between observed and predicted values and is the most informative in regard to the significance of the variables considered. With the exception of size all contextual variables are statistically significant. Income generation, processes improvement and intensity of partnerships are all positively associated with technical efficiency.
\end{abstract}

Keywords: DEA; bootstrap; two-stage estimation. 


\section{Introdução}

É de interesse para uma instituição pública de pesquisa ter informação de como aumentar sua produção com qualidade, sem demandar recursos adicionais. Uma melhor administração dos recursos disponíveis e a realização de padrões e objetivos pré-especificados são metas factíveis por meio do acompanhamento quantitativo do processo de produção. A Empresa Brasileira de Pesquisa Agropecuária (Embrapa) monitora, desde 1996, o processo de produção de seus 37 centros de pesquisa, por meio de um modelo de produção. Faz uso de uma fronteira determinística do tipo DEA (Data Envelopment Analysis), a qual permite avaliar a eficiência técnica de produção de cada um de seus centros de pesquisa. Crítico para o processo de produção é a identificação de unidades eficientes que funcionem como padrão para a organização e de variáveis contextuais, de controle gerencial, que influenciam positivamente a produção através da elevação do nível de eficiência técnica do sistema. De particular interesse no universo Embrapa são as variáveis intensidade de parcerias, geração de receita própria e melhoria de processos.

Em um contexto de ajuste de uma fronteira de produção não-paramétrica, ou de avaliação multicritério, via a Análise de Envoltória de Dados (DEA), em que o interesse está no estudo da significância estatística de um conjunto finito de variáveis exógenas na medida de eficiência técnica, tipicamente, faz-se uso de uma abordagem de regressão em dois estágios. Primeiramente estimam-se as medidas de eficiência técnica e, no segundo estágio, faz-se uso de um modelo de regressão para avaliar a significância dessas variáveis. Exemplos com este tipo de aplicação aparecem em Hoff (2006), Souza \& Staub (2007) e Souza et al. (2007). Essas aplicações envolvendo as regressões em dois estágios são complexas pois, por construção, as medidas de eficiência técnica são correlacionadas. Essa é uma das razões por que os procedimentos de regressão em dois estágios têm sido criticados na literatura recente. Veja-se Simar \& Wilson (2007). Quando o modelo de produção subjacente tem um único produto como resposta, Souza \& Staub (2007) mostram que a abordagem é válida assintoticamente. Simar \& Wilson (2007) mostram que a análise no caso geral (output com qualquer dimensão) é viável com o uso do bootstrap sob a hipótese adicional de separabilidade, isto é, desde que as variáveis contextuais sejam realmente exógenas e não alterem a fronteira de produção.

Nosso objetivo neste artigo é comparar as duas técnicas bootstrap propostas em Simar \& Wilson (2007) com um dos modelos de análise proposto em Souza, Staub \& Tabak (2006) sob a ótica do bootstrap e caracterizar a significância das variáveis contextuais de interesse no processo de produção da Embrapa para a melhor alternativa de modelagem.

A discussão levada a efeito no artigo procede como segue. Na Seção 2 introduz-se o sistema Embrapa de produção compreendendo as variáveis (insumos e produtos) e as variáveis contextuais consideradas de importância gerencial. A Seção 3 introduz o modelo de produção DEA. Na Seção 4 apresenta-se a análise estatística de modelos com resposta DEA, como o proposto em Souza, Staub \& Tabak (2006) e os bootstraps de Simar \& Wilson (2007). Na Seção 5 apresentam-se os resultados estatísticos e finalmente na Seção 6, apresentam-se as conclusões da análise e um resumo dos resultados obtidos. 


\section{O Sistema Embrapa de Produção}

A Empresa Brasileira de Pesquisa Agropecuária (Embrapa) é composta de 37 centros de pesquisa. Estes centros são classificados segundo suas missões e objetivos de pesquisa como centros ecorregionais (13), centros de produto (15) e centros temáticos (9). Também são classificados segundo sua estrutura de custos em pequenos (11), médios (18) e grandes (8).

A Embrapa monitora 28 variáveis de produto e 3 variáveis de insumo em seu sistema de produção de pesquisa. As variáveis de produção refletem atividades de produção técnico científica, de produção de publicações técnicas, de transferência de tecnologia e promoção da imagem e de desenvolvimento de tecnologias, produtos e processos. São agregadas em uma única medida de produção $(y)$ com a utilização de um sistema de pesos, variável por unidade, definidos pela administração superior da empresa. Cada variável de produção individualmente representa um índice adimensional que relaciona a quantidade produzida à média da instituição. Maiores detalhes sobre o sistema de pesos e os atributos considerados podem ser vistos em Souza, Avila \& Cruz (1997), Souza \& Ávila (1999) e Souza \& Avila (2000).

Os três insumos utilizados na caracterização da fronteira de produção são despesas de pessoal $\left(x_{1}\right)$, custeio $\left(x_{2}\right)$ e capital $\left(x_{3}\right)$ que representam proxies do quantum de uso desses insumos. Tal como o produto os insumos são definidos como escores adimensionais medidos em relação a média da empresa.

O uso de um produto univariado na análise torna as unidades de pesquisa da Embrapa comparáveis e viabiliza os resultados teóricos de Souza \& Staub (2007).

As variáveis de produção da Embrapa, consideradas para estudo neste artigo, são referentes ao ano de 2005 e estão apresentadas na Tabela 1 juntamente com as variáveis contextuais de interesse medidas no período. As variáveis contextuais são representadas por centros temáticos (Tipo T), de produto (Tipo P) e ecorregionais (Tipo E), de tamanho pequeno (Tam P), médio (Tam M) e grande (Tam G), receita própria (Recp), parcerias (Par) e melhoria de processos (Mproc). Os valores de Recp, Par e Mproc representam escores adimensionais, medidos em uma escala contínua, levantados pela empresa para cada centro de pesquisa. Esses construtos são de importância para o processo de produção pois fazem parte do indicador de desempenho institucional da empresa. Uma discussão detalhada das fórmulas de cálculo desses escores está disponível em Embrapa (2006). Os níveis de tamanho e tipo são variáveis indicadoras. A análise da covariável intensidade de parcerias é de particular importância pois permite detectar se a avaliação quantitativa da produção pode gerar competição nociva entre as unidades de pesquisa. 
Tabela 1 - Valores dos insumos $\left(x_{i}\right)$, produto agregado $(y)$ e variáveis contextuais tipo (Tipo), tamanho (Tam), receita própria (Recp), parcerias (Par) e melhoria de processos (Mproc), em 2005.

\begin{tabular}{|c|c|c|c|c|c|c|c|c|c|}
\hline Unidade & $x_{1}$ & $x_{2}$ & $x_{3}$ & $y$ & Tipo & Tam & Recp & Par & Mproc \\
\hline 1 & 2,045 & 3,216 & 2,133 & 3,244 & $\mathrm{~T}$ & G & 0,152 & 0,662 & 0,796 \\
\hline 2 & 0,934 & 0,857 & 0,796 & 0,849 & $\mathrm{P}$ & M & 0,789 & 0,295 & 0,000 \\
\hline 3 & 0,642 & 0,898 & 1,018 & 1,056 & $\mathrm{~T}$ & $\mathrm{M}$ & 0,122 & 0,688 & 0,503 \\
\hline 4 & 1,382 & 1,218 & 1,101 & 1,182 & $\mathrm{P}$ & $\mathrm{M}$ & 0,168 & 0,637 & 0,633 \\
\hline 5 & 1,074 & 1,629 & 1,333 & 1,105 & $\mathrm{~T}$ & M & 0,055 & 0,266 & 0,365 \\
\hline 6 & 0,682 & 0,668 & 0,852 & 0,417 & $\mathrm{P}$ & $\mathrm{P}$ & 0,074 & 0,447 & 0,540 \\
\hline 7 & 0,334 & 0,475 & 0,545 & 1,558 & $\mathrm{~T}$ & $\mathrm{P}$ & 0,276 & 0,577 & 0,775 \\
\hline 8 & 1,058 & 0,757 & 0,724 & 0,769 & $\mathrm{P}$ & M & 0,125 & 0,653 & 0,767 \\
\hline 9 & 1,037 & 0,952 & 3,495 & 0,555 & $\mathrm{P}$ & M & 0,166 & 0,512 & 0,000 \\
\hline 10 & 1,484 & 1,231 & 1,597 & 1,912 & $\mathrm{P}$ & G & 0,422 & 0,543 & 0,953 \\
\hline 11 & 0,997 & 1,195 & 0,824 & 1,226 & $\mathrm{P}$ & M & 0,076 & 0,513 & 0,804 \\
\hline 12 & 0,994 & 1,076 & 0,825 & 1,062 & $\mathrm{~T}$ & M & 0,084 & 0,615 & 0,918 \\
\hline 13 & 1,134 & 1,012 & 1,045 & 1,013 & $\mathrm{P}$ & M & 0,155 & 0,530 & 0,605 \\
\hline 14 & 1,645 & 1,765 & 0,804 & 1,286 & $\mathrm{P}$ & G & 0,222 & 0,734 & 0,486 \\
\hline 15 & 0,831 & 0,818 & 0,489 & 0,770 & $\mathrm{~T}$ & M & 0,325 & 0,405 & 0,419 \\
\hline 16 & 0,827 & 0,810 & 0,713 & 1,083 & $\mathrm{P}$ & M & 0,298 & 0,438 & 0,875 \\
\hline 17 & 1,512 & 1,887 & 1,083 & 1,797 & $\mathrm{P}$ & G & 1,000 & 0,512 & 0,618 \\
\hline 18 & 1,122 & 1,113 & 1,282 & 1,147 & $\mathrm{P}$ & M & 0,461 & 0,580 & 0,000 \\
\hline 19 & 0,487 & 0,472 & 1,044 & 1,757 & $\mathrm{~T}$ & $\mathrm{P}$ & 0,067 & 0,619 & 0,341 \\
\hline 20 & 0,706 & 1,042 & 0,409 & 0,959 & $\mathrm{P}$ & M & 0,294 & 0,204 & 0,721 \\
\hline 21 & 1,289 & 0,960 & 0,673 & 0,386 & $\mathrm{E}$ & G & 0,048 & 0,216 & 0,266 \\
\hline 22 & 1,761 & 1,385 & 1,270 & 0,858 & E & G & 0,261 & 0,430 & 0,900 \\
\hline 23 & 1,601 & 1,406 & 0,642 & 1,663 & $\mathrm{E}$ & G & 0,330 & 0,726 & 0,655 \\
\hline 24 & 0,624 & 0,662 & 0,589 & 0,265 & E & $\mathrm{P}$ & 0,067 & 0,613 & 0,459 \\
\hline 25 & 0,396 & 0,366 & 0,607 & 0,678 & E & $\mathrm{P}$ & 0,007 & 0,424 & 0,169 \\
\hline 26 & 0,640 & 0,537 & 0,456 & 1,190 & $\mathrm{E}$ & $\mathrm{P}$ & 0,057 & 0,549 & 0,577 \\
\hline 27 & 0,480 & 0,584 & 0,933 & 0,402 & E & $\mathrm{P}$ & 0,074 & 0,261 & 0,253 \\
\hline 28 & 1,092 & 0,787 & 1,319 & 0,583 & E & M & 0,184 & 0,465 & 1,000 \\
\hline 29 & 0,574 & 0,583 & 1,003 & 0,761 & E & $\mathrm{P}$ & 0,092 & 1,000 & 0,822 \\
\hline 30 & 0,620 & 0,503 & 0,782 & 0,794 & E & $\mathrm{P}$ & 0,022 & 0,501 & 0,273 \\
\hline 31 & 0,893 & 0,876 & 1,273 & 0,939 & E & $\mathrm{M}$ & 0,098 & 0,340 & 0,666 \\
\hline 32 & 1,297 & 1,048 & 1,347 & 0,697 & E & $\mathrm{M}$ & 0,150 & 0,262 & 0,743 \\
\hline 33 & 2,651 & 1,989 & 1,100 & 1,537 & E & G & 0,162 & 0,210 & 0,588 \\
\hline 34 & 0,710 & 0,458 & 0,892 & 0,838 & $\mathrm{P}$ & $\mathrm{M}$ & 0,235 & 0,884 & 0,827 \\
\hline 35 & 0,441 & 0,399 & 0,606 & 0,153 & $\mathrm{P}$ & $\mathrm{P}$ & 0,065 & 0,663 & 0,000 \\
\hline 36 & 0,763 & 0,836 & 0,588 & 1,206 & $\mathrm{~T}$ & M & 0,194 & 0,393 & 0,756 \\
\hline 37 & 0,242 & 0,528 & 0,805 & 0,174 & $\mathrm{~T}$ & $\mathrm{P}$ & 0,107 & 0,089 & 0,463 \\
\hline
\end{tabular}




\section{Análise de Envoltória de Dados (DEA)}

Considere um processo produtivo constituído de $n$ unidades de produção, usualmente denominadas em DEA de DMU's (Decision Making Units). A DMU de índice $k$, $k=1, \ldots, n$, se utiliza do vetor de quantidades $x_{k}$ de $s$ insumos, com componentes não negativas e não todas nulas, na produção do vetor de quantidades $y_{k}$, de dimensão $r$, com componentes não negativas e não todas nulas. Denote por $Y=\left(y_{1}, y_{2}, \ldots, y_{n}\right)$ a matriz de produção $r \times n$ e por $X=\left(x_{1}, x_{2}, \ldots, x_{n}\right)$ a matriz $s \times n$ de utilização de insumos. Assim, para uma dada unidade $k$ com vetor de produção $\left(x_{k}, y_{k}\right)$, a medida de eficiência técnica $\hat{\theta}_{k}$ é obtida resolvendo o seguinte problema de programação linear:

$$
\hat{\theta}_{k}=\min \left\{\theta ; y_{k} \leq \sum_{i=1}^{n} \gamma_{i} y_{i} ; \theta x_{k} \geq \sum_{i=1}^{n} \gamma_{i} x_{i} ; \theta>0 ; \gamma_{i} \geq 0, i=1, \ldots, n\right\} .
$$

Se $\hat{\theta}_{k}=1$, a DMU $k$ é considerada tecnicamente eficiente.

A formulação acima do cálculo da medida DEA de eficiência pressupõe retornos constantes à escala e é orientada para insumos. Veja Coelli et al. (2005). A solução com retornos variáveis é obtida adicionando-se a restrição $\sum_{i=1}^{n} \gamma_{i}=1$.

A Embrapa se utiliza da medida DEA, com orientação a insumos, sob a suposição de retornos constantes à escala, no cálculo da eficiência técnica de produção de cada um dos seus centros de pesquisa. A hipótese de retornos constantes se justifica em parte pelo uso de um sistema de pesos diferenciados no cálculo das medidas combinadas de output e por testes estatísticos anteriores (Souza, 2001, 2003). Neste contexto se torna particularmente conveniente a avaliação da significância estatística da variável contextual tamanho na medida de eficiência.

\section{Análise Estatística de Modelos com Resposta DEA}

Os modelos de regressão em dois estágios, associados à medida de eficiência técnica, tipicamente, especificam a distribuição da medida de eficiência estimada (no primeiro estágio) como dependente do construto linear $\mu=z^{\prime} \beta$, sendo $z$ o vetor de observação nas variáveis contextuais, de dimensão $p$, e $\beta$ um parâmetro $p$-dimensional desconhecido. Souza, Staub \& Tabak (2006) consideram várias alternativas de modelagem para a distribuição de $\hat{\theta}$ incluindo os modelos do tipo Tobit. As formulações que consideraremos aqui se baseiam na distribuição $N\left(\mu, \sigma^{2}\right)$ truncada no intervalo $(0,1)$. A função densidade de probabilidades dessa variável aleatória vem dada por:

$$
f(\varepsilon)=\left\{\begin{array}{cc}
\frac{1}{\sigma} \phi\left(\frac{\varepsilon-\mu}{\sigma}\right) /\left(\Phi\left(\frac{1-\mu}{\sigma}\right)-\Phi\left(\frac{-\mu}{\sigma}\right)\right), & \text { se } 0<\varepsilon<1 \\
0, & \text { c.c. }
\end{array}\right.
$$

As funções $\phi($.$) e \Phi($.$) representam, respectivamente, as funções de densidade e de distri-$ buição de probabilidades da normal padrão.

Pesquisa Operacional, v.29, n.2, p.289-302, Maio a Agosto de 2009 
$O$ vetor de parâmetros $\beta$ de efeitos técnicos presentes em $\mu$ é estimado pela maximização da função de verossimilhança conjunta das $n$ medidas de eficiência:

$$
L(\beta, \sigma)=\prod_{k=1}^{n} \frac{\phi\left(\frac{x_{k}-\mu_{k}}{\sigma}\right)}{\sigma\left[\Phi\left(\frac{1-\mu_{k}}{\sigma}\right)-\Phi\left(\frac{-\mu_{k}}{\sigma}\right)\right]} .
$$

Notamos que a distribuição normal truncada em $(0,1)$ pode ser caracterizada através da representação

$$
\hat{\theta}_{k}=\mu_{k}+\varepsilon_{k} \quad\left(\mu_{k}=z_{k}^{\prime} \beta\right)
$$

onde a variável aleatória $\varepsilon_{k}$ é a normal $N\left(0, \sigma^{2}\right)$ truncada à esquerda em $-\mu_{k}$ e à direita em $1-\mu_{k}$.

Os problemas de estimação envolvidos no ajuste do modelo de regressão (4), como observado em Simar \& Wilson (2007), são de três tipos. Correlação serial, viés em $\hat{\theta}_{k} \mathrm{e}$ correlação das variáveis contextuais com os erros $\varepsilon_{k}$. Sob certas condições de regularidade, para os modelos de produção com um único output, Banker (1993) e Souza \& Staub (2007) mostram que a análise se justifica assintoticamente. Banker \& Natarajan (2004) e Souza, Staub \& Tabak (2006) também sugerem modelos heurísticos para o ajuste de medidas DEA associadas a modelos de produção com outputs múltiplos. No contexto de Souza \& Staub (2007) o procedimento bootstrap também pode ser utilizado para corrigir o viés e os desvios padrão dos estimadores dos parâmetros $\beta$ e $\sigma$.

No caso geral, sob um conjunto de condições de regularidade diferente, Simar \& Wilson (2007) propõem dois procedimentos alternativos do tipo bootstrap, apresentados abaixo em forma de Algoritmos (um e dois). O primeiro procedimento destina-se a melhorar a inferência estatística das medidas de eficiência técnica. O segundo procura fazer o mesmo, com redução concomitante do viés do estimador em pequenas amostras.

O bootstrap do Algoritmo 1 só difere do bootstrap associado ao modelo proposto por Souza, Staub \& Tabak (2006) na eliminação das observações com eficiência unitária.

Observamos que as três abordagens bootstrap exigem realizações iid da distribuição $N\left(\mu, \sigma^{2}\right)$ truncada em $(0,1)$. Conhecidos $\mu$ e $\sigma$ essas realizações são geradas utilizando a expressão

$$
t=\mu+\sigma \Phi^{-1}\left[w \Phi\left(\frac{1-\mu}{\sigma}\right)+\Phi\left(\frac{-\mu}{\sigma}\right)(1-w)\right]
$$

onde $w$ é uma variável aleatória com distribuição uniforme em $(0,1)$.

Iniciamos agora a descrição dos métodos bootstrap que serão utilizados em nossa análise.

\section{Algoritmo 1 (Simar \& Wilson, 2007):}

1. Para cada $\left(x_{k}, y_{k}\right), k=1, \ldots, n$, calcule $\hat{\theta}_{k}$ usando (1);

2. Use o método de máxima verossimilhança para obter um estimador $\hat{\beta}$ de $\beta$, bem como um estimador $\hat{\sigma}$ de $\sigma$ na regressão normal truncada de $\hat{\theta}_{k}$ em $z_{k}^{\prime} \beta$ em (4), 
usando $m<n$ observações quando $\hat{\theta}_{k}<1$, isto é, eliminando as observações com eficiência unitária;

3. Repita os passos (3.1-3.3) abaixo $L$ vezes para obter o conjunto de repetições bootstrap $A=\left\{\left(\hat{\beta}^{*}, \hat{\sigma}^{*}\right)_{b}\right\}_{b=1}^{L}$.

3.1. Para $k=1, \ldots, m$, gere $\varepsilon_{k}$ da distribuição $N\left(0, \hat{\sigma}^{2}\right)$ truncada à esquerda em $-z_{k} \hat{\beta}$ e à direita em $1-z_{k} \hat{\beta}$;

3.2. Para $k=1, \ldots, m$ calcule $\theta_{k}^{*}=z_{k}^{\prime} \hat{\beta}+\varepsilon_{k}^{*}$;

3.3. Use o método de máxima verossimilhança para estimar a regressão de $\theta_{k}^{*}$ em $z_{k}$ (4), obtendo novas estimativas $\left(\hat{\beta}^{*}, \hat{\sigma}^{*}\right)$;

4. Use as repetições bootstrap em $A$ e os estimadores originais $\hat{\beta}, \hat{\sigma}$ para obter estimativas bootstrap de parâmetros, desvios padrão e intervalos de confiança.

$\mathrm{O}$ viés de $\hat{\theta}_{k}$, relativamente ao valor verdadeiro $\theta_{k}$ da eficiência técnica,

$$
\operatorname{vies}\left(\hat{\theta}_{k}\right)=E\left(\hat{\theta}_{k}\right)-\theta_{k}
$$

é estimado por

$$
\widehat{\operatorname{vies}}\left(\hat{\theta}_{k}\right)=E\left(\hat{\theta}_{k}^{*}\right)-\hat{\theta}_{k},
$$

onde o valor esperado $E\left(\hat{\theta}_{k}\right)$ é aproximado por meio da média das realizações $\hat{\theta}_{k, b}^{*}$. Portanto,

$$
\widehat{\operatorname{vies}}\left(\hat{\theta}_{k}\right)=\frac{1}{L} \sum_{b=1}^{L} \hat{\theta}_{k, b}^{*}-\hat{\theta}_{k}=\bar{\theta}_{k}^{*}-\hat{\theta}_{k} .
$$

Assim, um estimador com viés corrigido de $\theta_{k}$ é:

$$
\tilde{\theta}_{k}=\hat{\theta}_{k}-\widehat{\operatorname{vies}}\left(\hat{\theta}_{k}\right)=2 \hat{\theta}_{k}-\bar{\theta}_{k}^{*} \text {. }
$$

A seguir apresenta-se o segundo algoritmo.

\section{Algoritmo 2 (Simar \& Wilson, 2007):}

1. Para cada $\left(x_{k}, y_{k}\right), k=1, \ldots, n$, calcule $\hat{\theta}_{k}$ usando (1);

2. Use o método de máxima verossimilhança para obter um estimador $\hat{\beta}$ de $\beta$, bem como um estimador $\hat{\sigma}$ de $\sigma$ na regressão normal truncada de $\hat{\theta}_{k}$ em $z_{k}$ em (4), usando $m<n$ observações quando $\hat{\theta}_{k}<1$, isto é, elimine as observações com eficiência unitária;

3. Repita os passos (3.1-3.4) $L_{1}$ vezes para obter os $n$ conjuntos de repetições bootstrap $B_{k}=\left\{\hat{\theta}_{k b}^{*}\right\}_{b=1}^{L_{1}}$. 
3.1. Para $k=1, \ldots, n$, gere $\varepsilon_{k}$ da distribuição $N\left(0, \hat{\sigma}^{2}\right)$ truncada à esquerda em $-z_{k b} \hat{\beta}$ e à direita em $1-z_{k b} \hat{\beta}$.

3.2. Para $k=1, \ldots, n$. Calcule $\theta_{k b}^{*}=z_{k b} \hat{\beta}+\varepsilon_{k b}^{*}$;

3.3. Calcule $x_{k b}^{*}=\left(\hat{\theta}_{k} / \theta_{k b}^{*}\right) x_{k}$, para todo $k=1, \ldots, n$. Esta correção dos insumos é necessária e é herdada de Simar \& Wilson (1998);

3.4. Calcule o estimador bootstrap $\hat{\theta}_{k b}^{*}$ de $\hat{\theta}_{k}$, para $k=1, \ldots, n$, resolvendo $\hat{\theta}_{k b}^{*}=\min \left\{\theta ; y_{k} \leq \sum_{i=1}^{n} \gamma_{i} y_{i} ; \theta x_{k} \geq \sum_{i=1}^{n} \gamma_{i} x^{*}{ }_{k b} ; \theta>0 ; \gamma_{i} \geq 0, i=1, \ldots, n\right\}$.

4. Para $k=1, \ldots, n$, calcule o estimador viés corrigido de $\tilde{\theta}_{k}$, como em (9).

5. Use o método de máxima verossimilhança para estimar a regressão normal truncada de $\tilde{\theta}_{k}$ em $z_{k}$, obtendo as estimativas $(\tilde{\beta}, \tilde{\sigma})$.

6. Repita os passos (6.1-6.3) $L_{2}$ vezes para obter um conjunto de repetições bootstrap $C=\left\{\left(\hat{\beta}^{*}, \hat{\sigma}_{\varepsilon}^{*}\right)_{b}\right\}_{b=1}^{L_{2}}$.

6.1. Para $k=1, \ldots, n$, gere $\varepsilon_{k}$ da distribuição $N(0, \tilde{\sigma})$ truncada à esquerda em $-z_{k} \tilde{\beta}$ e à direita em $1-z_{k} \tilde{\beta}$;

6.2. Para $k=1, \ldots, n$ calcule $\theta_{k}^{* *}=z_{k} \tilde{\beta}+\varepsilon_{k}$;

6.3. Use o método de máxima verossimilhança para estimar a regressão truncada de $\theta_{k}^{* *}$ em $z_{k}$, obtendo as estimativas $\left(\hat{\beta}^{*}, \hat{\sigma}^{*}\right)$;

7. Use as repetições bootstrap em $C$ e as estimativas originais $(\tilde{\beta}, \tilde{\sigma})$ na obtenção de estimativas bootstrap dos parâmetros, desvios padrão e intervalos de confiança.

Os passos 1 e 2 no Algoritmo 2 são os mesmos que no Algoritmo 1. Os passos 3 e 4 no Algoritmo 2 usam um bootstrap paramétrico no problema do primeiro estágio para produzir estimativas do viés corrigido $\tilde{\theta}_{k}$. Basicamente, os passos 5 e 6 não diferem no Algoritmo 1, com exceção de $\tilde{\theta}_{k}$ substituir $\theta_{k}$ em (4) em vez de $\hat{\theta}_{k}$, como no Algoritmo 1 . Uma vez que o conjunto de valores bootstrap $A$ ou $C$ seja obtido, como no passo 3 do Algoritmo 1, ou como no passo 6 do Algoritmo 2, os intervalos de confiança bootstrap podem ser construídos.

A presença de viés apreciável, em qualquer caso, nas distribuições bootstrap dos parâmetros deteriora a performance do intervalo de confiança percentil simples. Para corrigir esse problema, utilizou-se o intervalo de confiança percentil viés corrigido. Veja Souza (1998).

As escolhas de $L$, no primeiro algoritmo e $L_{2}$, no segundo, determinam o número de repetições bootstrap usadas para construir os intervalos de confiança nos dois algoritmos. Simar \& Wilson (2007) usam 2000 repetições. A escolha de $L_{1}$, no segundo algoritmo, determina o número de repetições bootstrap usadas para calcular os estimadores do viés corrigido $\tilde{\theta}$. Segundo Simar \& Wilson (2007) 100 repetições são suficientes para esta finalidade. 
O pacote estatístico SAS v.9.1 foi usado tanto para calcular o estimador de eficiência DEA, como para estimar os modelos de regressão truncada. O Algoritmo 2, por ser mais complexo que o Algoritmo 1, exige uma maior demanda computacional para ser executado.

\section{Resultados Estatísticos}

Discutimos agora os resultados da aplicação dos três métodos do tipo bootstrap aos dados da Tabela 1. Iniciamos com a técnica de máxima verossimilhança aplicada a todo o conjunto de observações. Essas estimativas constam da Tabela 2. Na Tabela 3 apresentam-se os resultados que se obtém para o estimador de máxima verossimilhança eliminando-se as observações com eficiência unitária.

Tabela 2 - Ajuste de máxima verossimilhança, dist. normal $N(\mu, \sigma)$ truncada em $(0,1)$, com todas as observações. Os intervalos de confiança (IC) são assintóticos e no nível de $95 \%$.

\begin{tabular}{lcccccc}
\hline Parâmetro & Estimativa & $\begin{array}{c}\text { Desvio } \\
\text { Padrão }\end{array}$ & $t$ & $\operatorname{Pr}>|t|$ & $\begin{array}{c}\text { IC } \\
\text { (lim inf) }\end{array}$ & $\begin{array}{c}\text { IC } \\
\text { (lim sup) }\end{array}$ \\
\hline Intercepto & 0,060 & 0,201 & 0,3 & 0,767 & $-0,347$ & 0,467 \\
Recp & 0,525 & 0,339 & 1,55 & 0,130 & $-0,162$ & 1,211 \\
Par & 0,295 & 0,280 & 1,05 & 0,300 & $-0,273$ & 0,863 \\
Mproc & 0,310 & 0,214 & 1,45 & 0,155 & $-0,123$ & 0,743 \\
Tipo T & 0,233 & 0,144 & 1,62 & 0,115 & $-0,059$ & 0,525 \\
Tipo P & $-0,016$ & 0,141 & $-0,11$ & 0,909 & $-0,301$ & 0,269 \\
Tam M & $-0,180$ & 0,144 & $-1,25$ & 0,219 & $-0,473$ & 0,112 \\
Tam G & $-0,116$ & 0,174 & $-0,66$ & 0,510 & $-0,468$ & 0,237 \\
s2 & 0,063 & 0,025 & 2,5 & 0,017 & 0,012 & 0,114 \\
\hline
\end{tabular}

Tabela 3 - Ajuste de máxima verossimilhança, dist. normal $N(\mu, \sigma)$ truncada em $(0,1)$, excluindo eficiências iguais a 1 . Os intervalos de confiança (IC) são assintóticos e no nível de $95 \%$.

\begin{tabular}{lcccccc}
\hline Parâmetro & Estimativa & $\begin{array}{c}\text { Desvio } \\
\text { Padrão }\end{array}$ & $t$ & $\operatorname{Pr}>|t|$ & $\begin{array}{c}\text { IC } \\
\text { (lim Inf) }\end{array}$ & $\begin{array}{c}\text { IC } \\
\text { (lim sup) }\end{array}$ \\
\hline Intercepto & 0,118 & 0,142 & 0,83 & 0,413 & $-0,171$ & 0,407 \\
Recp & 0,321 & 0,241 & 1,33 & 0,191 & $-0,168$ & 0,811 \\
Par & 0,181 & 0,209 & 0,87 & 0,391 & $-0,242$ & 0,605 \\
Mproc & 0,220 & 0,157 & 1,4 & 0,170 & $-0,099$ & 0,540 \\
Tipo T & 0,037 & 0,113 & 0,33 & 0,746 & $-0,193$ & 0,267 \\
Tipo P & $-0,042$ & 0,107 & $-0,4$ & 0,694 & $-0,259$ & 0,174 \\
Tam M & 0,005 & 0,117 & 0,04 & 0,967 & $-0,232$ & 0,241 \\
Tam G & 0,047 & 0,135 & 0,35 & 0,731 & $-0,228$ & 0,321 \\
s2 & 0,042 & 0,014 & 3,09 & 0,004 & 0,014 & 0,069 \\
\hline
\end{tabular}

Nos dois modelos não há indicação de relevância da presença das variáveis contextuais. A correção via bootstrap altera este resultado para a proposta que não exclui as unidades eficientes. 
A Tabela 4 mostra os resultados que encontramos para o Algoritmo 1. Existem instâncias onde o viés relativo é apreciável, isto é, maior que $1 \%$.

Os parâmetros com comportamento excessivamente não-linear são o Intercepto, Mproc, Tipo $\mathrm{T}$, Tam $\mathrm{G}$ e $\sigma^{2}$. A distribuição dos estimadores dos parâmetros também se afasta consideravelmente da normal sugerindo que os intervalos de confiança constantes da Tabela 3 tais como os da tabela 2 não estão corretos.

Tabela 4 - Resultados para o Algoritmo 1: médias e desvios padrão das repetições bootstrap, teste de normalidade de Kolmogorov-Smirnov, estimativa do parâmetro com correção do viés e viés relativo.

\begin{tabular}{lcccccc}
\hline Parâmetro & $\begin{array}{c}\text { Média } \\
\text { Rep. }\end{array}$ & $\begin{array}{c}\text { Desvio } \\
\text { Padrão }\end{array}$ & $K S$ & $\begin{array}{c}\mathrm{p} \mathrm{Valor} \\
(\mathrm{KS})\end{array}$ & $\begin{array}{c}\text { Param. } \\
\text { Viés Corr. }\end{array}$ & $\begin{array}{c}\text { Viés } \\
\text { Relativo (\%) }\end{array}$ \\
\hline Intercepto & 0,124 & 0,141 & 0,031 & $<0,01$ & 0,112 & 5,08 \\
Recp & 0,32 & 0,246 & 0,021 & $<0,01$ & 0,323 & 0,31 \\
Par & 0,181 & 0,207 & 0,017 & 0,031 & 0,181 & 0,00 \\
Mproc & 0,217 & 0,158 & 0,023 & $<0,01$ & 0,224 & 1,36 \\
Tipo T & 0,036 & 0,112 & 0,022 & $<0,01$ & 0,038 & 2,70 \\
Tipo P & $-0,042$ & 0,104 & 0,012 & $>0,15$ & $-0,042$ & 0,00 \\
Tam M & 0,004 & 0,116 & 0,016 & 0,070 & 0,006 & 20,00 \\
Tam G & 0,045 & 0,135 & 0,02 & $<0,01$ & 0,049 & 4,26 \\
s2 & 0,032 & 0,012 & 0,093 & $<0,01$ & 0,052 & 23,81 \\
\hline
\end{tabular}

Os resultados para o Algoritmo 2 constam da Tabela 5. As estimativas diferem das do Algoritmo 1 com uma mudança de sinal. Normalidade é aceitável para a maioria dos parâmetros.

Tabela 5 - Resultados para o Algoritmo 2: Estimativas e desvios padrão bootstrap e teste de normalidade de Kolmogorov-Smirnov.

\begin{tabular}{lcccc}
\hline \multicolumn{1}{c}{ Parâmetro } & Estim. Bootstrap & Desvio Padrão & $K S$ & p valor $(\mathrm{KS})$ \\
\hline Intercepto & 0,044 & 0,129 & 0,028 & $<0,01$ \\
Recp & 0,264 & 0,205 & 0,020 & 0,050 \\
Par & 0,125 & 0,185 & 0,013 & $>0,15$ \\
Mproc & 0,185 & 0,137 & 0,019 & 0,078 \\
Tipo T & 0,071 & 0,090 & 0,009 & $>0,15$ \\
Tipo P & $-0,016$ & 0,094 & 0,014 & $>0,15$ \\
Tam M & $-0,023$ & 0,094 & 0,018 & 0,098 \\
Tam G & 0,049 & 0,111 & 0,016 & $>0,15$ \\
s2 & 0,024 & 0,009 & 0,080 & $<0,01$ \\
\hline
\end{tabular}

Para as repetições bootstrap incluindo as medidas de eficiência unitária obtêm-se os resultados da Tabela 6 . Valem aqui considerações similares às feitas anteriormente para o Algoritmo 1, sobre o viés e a normalidade dos estimadores, isto é, as distribuições booststrap são não normais. 
Tabela 6 - Resultados para o bootstrap com todas as observações: médias e desvio padrão das repetições bootstrap, teste de normalidade de Kolmogorov-Smirnov, estimativa do parâmetro com correção do viés e viés relativo.

\begin{tabular}{lcccccc}
\hline Parâmetro & $\begin{array}{c}\text { Média } \\
\text { Rep. }\end{array}$ & $\begin{array}{c}\text { Desvio } \\
\text { Padrão }\end{array}$ & $K S$ & $\begin{array}{c}\text { p valor } \\
(\mathrm{KS})\end{array}$ & $\begin{array}{c}\text { Param. Viés } \\
\text { Corr. }\end{array}$ & $\begin{array}{c}\text { Viés Relativo } \\
(\%)\end{array}$ \\
\hline Intercepto & 0,069 & 0,204 & 0,059 & $<0,01$ & 0,050 & 15,00 \\
Recp & 0,521 & 0,345 & 0,058 & $<0,01$ & 0,528 & 0,76 \\
Par & 0,291 & 0,281 & 0,029 & $<0,01$ & 0,299 & 1,35 \\
Mproc & 0,304 & 0,216 & 0,035 & $<0,01$ & 0,316 & 1,93 \\
Tipo T & 0,227 & 0,144 & 0,053 & $<0,01$ & 0,240 & 2,57 \\
Tipo P & $-0,016$ & 0,142 & 0,021 & $<0,01$ & $-0,016$ & 0,00 \\
Tam M & $-0,178$ & 0,147 & 0,043 & $<0,01$ & $-0,183$ & 1,11 \\
Tam G & $-0,114$ & 0,174 & 0,026 & $<0,01$ & $-0,117$ & 1,72 \\
s2 & 0,049 & 0,025 & 0,116 & $<0,01$ & 0,076 & 22,22 \\
\hline
\end{tabular}

A significância das variáveis contextuais é analisada a partir dos intervalos de confiança viés corrigidos. Esses constam da Tabela 7 para as três alternativas estudadas. Não há evidência de significância no contexto dos Algoritmos 1 e 2 . A inclusão de todas as observações muda substancialmente esse resultado, particularmente com respeito ao Algoritmo 1. Nesse contexto as variáveis Recp (receita própria), Par (parcerias) e Mproc (melhoria de processos) têm efeito positivo e significativo na medida de eficiência.

Tabela 7 - Intervalos de confiança viés corrigidos para o Algoritmo 1 (L A1, U A1), para o modelo com todas as observações (L M, U M) e para o Algoritmo 2 (L A2, U A2).

\begin{tabular}{lcccccc}
\hline \multicolumn{1}{c}{ Parâmetro } & L A1 & U A1 & L M & U M & L A2 & U A2 \\
\hline Intercepto & $-0,207$ & 0,351 & $-0,071$ & 0,562 & $-0,231$ & 0,274 \\
Recp & $-0,126$ & 0,855 & 0,345 & 2,528 & $-0,135$ & 0,672 \\
Par & $-0,208$ & 0,609 & 0,146 & 1,926 & $-0,234$ & 0,487 \\
Mproc & $-0,050$ & 0,573 & 0,198 & 2,316 & $-0,073$ & 0,462 \\
Tipo T & $-0,159$ & 0,272 & 0,164 & 1,951 & $-0,103$ & 0,250 \\
Tipo P & $-0,239$ & 0,164 & $-0,099$ & 0,673 & $-0,205$ & 0,165 \\
Tam M & $-0,210$ & 0,243 & $-0,260$ & 0,275 & $-0,210$ & 0,165 \\
Tam G & $-0,218$ & 0,314 & $-0,214$ & 0,459 & $-0,173$ & 0,264 \\
s2 & 0,029 & 0,121 & 0,056 & 0,375 & 0,012 & 0,047 \\
\hline
\end{tabular}

Com o intuito de avaliar com mais detalhes os ajustes dos modelos calculamos uma medida de adequabilidade. A medida escolhida foi o coeficiente de correlação de Pearson entre os valores observados da eficiência técnica e os valores preditos pelos modelos. Os valores encontrados para o coeficiente de correlação foram $0,40,0,42$ e 0,50 para o Algoritmo 1, Algoritmo 2 e para o estimador de máxima verossimilhança, com todas as observações, corrigidos pelo viés, respectivamente.

A evidência não é de ajuste excelente para nenhum dos três modelos, mas a alternativa que inclui todas as observações é superior posto que: 
1. É a única alternativa consoante com as expectativas de sinais dos coeficientes.

2. É a única alternativa que conduz a significância das variáveis contextuais via bootstrap. Tal significância depreende-se do processo de avaliação da Embrapa e é esperada em pelo menos dois dos atributos contínuos.

3. O acréscimo de $8 \%$ na correlação de Pearson é significante. Deste modo o estimador de máxima verossimilhança viés corrigido incluindo todas as observações define o procedimento que reproduz mais fidedignamente as variações nos valores da resposta. Note que para o Algoritmo 1, as eficiências unitárias são reduzidas para perto de 0,4 e 0,5 e no Algoritmo 2 para perto de 0,3 e 0,4. Esses resultados não são aceitáveis. As Figuras 1,2 e 3 ilustram o fato.

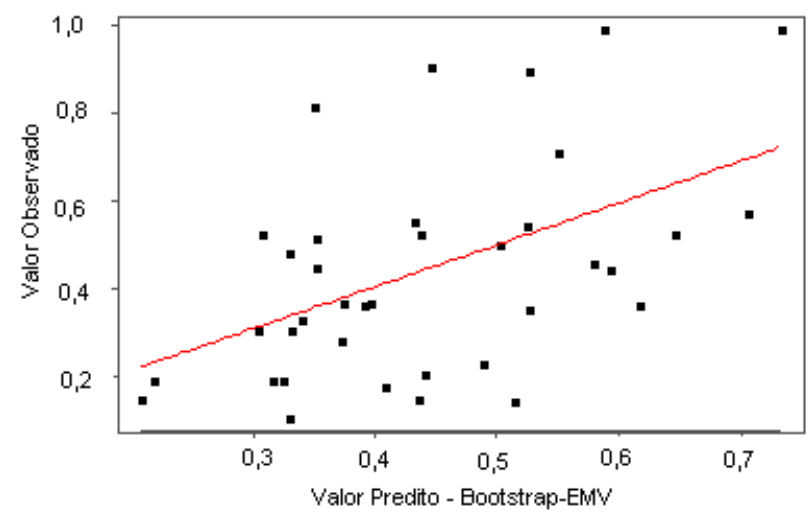

Figura 1 - Gráfico de valores observados vs valores preditos. Bootstrap do EMV com todas as observações:

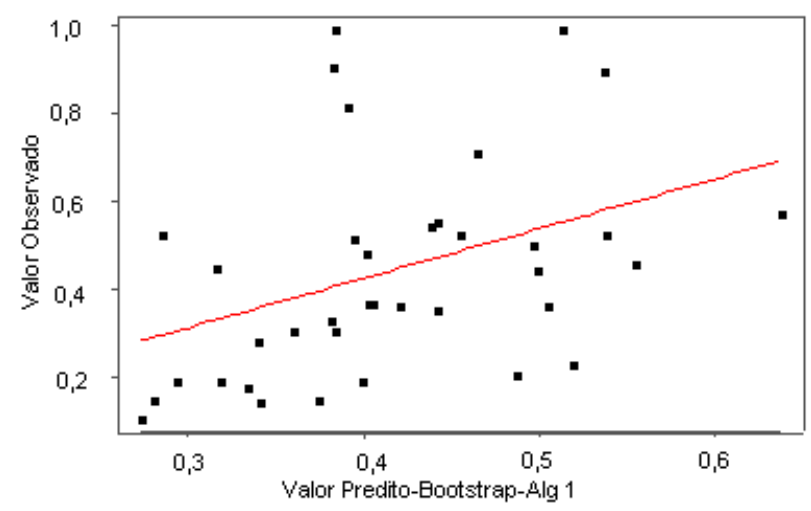

Figura 2 - Gráfico de valores observados vs valores preditos. Algoritmo 1. 


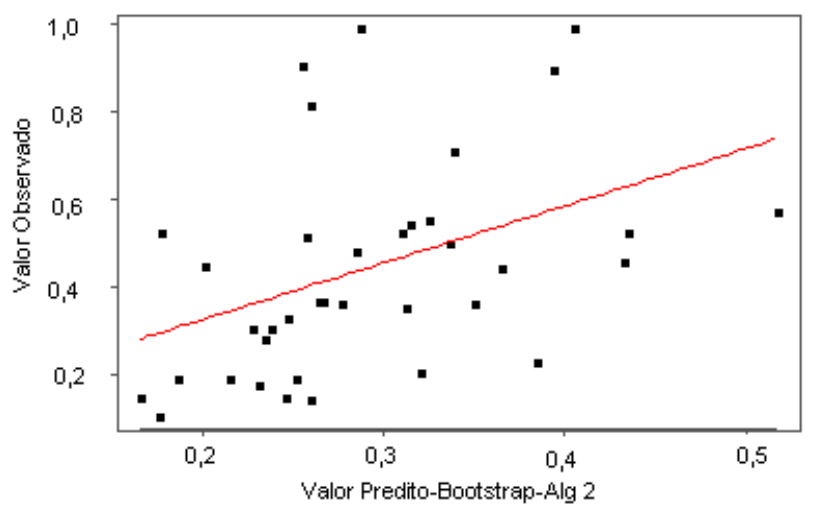

Figura 3 - Gráfico de valores observados vs valores preditos. Algoritmo 2.

\section{Resumo e Conclusões}

Apresentamos no artigo três alternativas de métodos bootstrap para a avaliação da significância de covariáveis em modelos estatísticos nos quais se estuda o efeito dessas variáveis em uma medida de eficiência técnica calculada com o uso de Análise de Envoltória de Dados. A dificuldade da análise desses modelos reside na presença de correlação e viés nas medidas DEA e de correlação das variáveis contextuais com os resíduos do modelo. Os dois algoritmos propostos por Simar \& Wilson (2007) foram comparados entre si e com o método proposto por Souza, Staub \& Tabak (2006). A aplicação de interesse em que os métodos foram aplicados consiste na determinação da importância das variáveis intensidade da captação de recursos para a pesquisa, intensidade de parcerias, melhoria de processos administrativos, tipo e tamanho em uma medida de eficiência técnica DEA, orientada para insumos e calculada, sob a hipótese de retornos constantes à escala, para cada um dos 37 centros de pesquisa da Embrapa. Conclui-se do exercício estatístico que o modelo de Souza, Staub \& Tabak (2006) mostrou-se mais adequado para a aplicação no sentido de ajustar-se melhor às observações do ponto de vista da correlação entre valores preditos e observados e mais informativo em detectar a significância das variáveis contextuais, resultado intuitivamente esperado. Nesse contexto, conclui-se que todas as variáveis contextuais são significantes e positivamente associadas à medida de eficiência.

\section{Referências Bibliográficas}

(1) Banker, R.D. (1993). Maximum Likelihood Consistency and DEA: a Statistical Foundation. Management Science, 39(10), 1265-1273.

(2) Banker, R.D. \& Natarajan, R. (2004). Statistical Tests Based on DEA Efficiency Scores. Handbook of Data Envelopment Analysis, Kluwer, New York.

(3) Coelli, T.; Rao, D.S.P.; O’Donnel, C.J. \& Battese, G.E. (2005). An Introduction to Efficiency and Productivity Analysis. $2^{\text {nd }}$ edition, Springer, NY.

(4) Embrapa. (2006). Manual de Indicadores de avaliação de desempenho das unidades descentralizadas da Embrapa: Metas quantitativas - Versão para ano base 2007. Brasília: Superintendência de Pesquisa e Desenvolvimento. 
(5) Hoff, A. (2006). Second stage DEA: Comparison of approaches for modelling the DEA score. European Journal of Operational Research, 181(3), 425-435.

(6) Simar, L. \& Wilson P. (1998). Sensibility Analysis of Efficiency Scores: How to Bootstrap in Nonparametric Frontier Models. Management Science, 44(1), 49-61.

(7) Simar, L. \& Wilson P. (2007). Estimation and Inference in Two-Stage, Semi-Parametric Models of Production Process. Journal of Econometrics, 136, 31-64.

(8) Souza, G.S.; Alves, E.; Ávila, A.F.D. \& Cruz, E.R. (1997). Produtividade e Eficiência Relativa de Produção em Sistemas de Produção de Pesquisa Agropecuária. Revista Brasileira de Economia, 51(3), 281-307.

(9) Souza, G.S. (1998). Introdução aos Modelos de Regressão Linear e Não-Linear. Embrapa, Brasília.

(10) Souza, G.S.; Alves, E. \& Ávila, A.F.D. (1999). Technical Efficiency in Agricultural Research. Scientometrics, 46, 141-160.

(11) Souza, G.S. \& Ávila, A.F.D. (2000). A Psicometria Linear da Escalagem Ordinal: uma Aplicação na Caracterização da Importância Relativa de Atividades de Produção em Ciência e Tecnologia. Cadernos de Ciência e Tecnologia, 17(3), 11-27.

(12) Souza, G.S. (2001). Statistical properties of nonparametric estimators of DEA estimators of production frontiers. Revista de Econometria, 21(2), 291-322.

(13) Souza, G.S. (2003). Funções de produção: Uma abordagem estatística com o uso de modelos de encapsulamento de dados. Texto para Discussão, Brasília, 17, 8-49.

(14) Souza, G.S.; Staub, R.B. \& Tabak, B. (2006). Assessing the Significance of Factors Effects in Output Oriented DEA Measures of Efficiency: an Application to Brazilian Banks. Revista Brasileira de Economia de Empresas, 6, 7-20.

(15) Souza, G.S. \& Staub, R.B. (2007). Two Stage Inference Using DEA Efficiency Measurements in Univariate Production Models. International Transactions of Operations Research, 14, 15-26.

(16) Souza, G.S.; Gomes, E.G.; Magalhães, M.C. \& Ávila, A.F.D. (2007). Economic efficiency of Embrapa's research centers and the influence of contextual variables. Pesquisa Operacional, 27(1), 15-26. 